\title{
Measurement of photon production in the very forward direction in deep-inelastic scattering at HERA
}

The H1 Collaboration

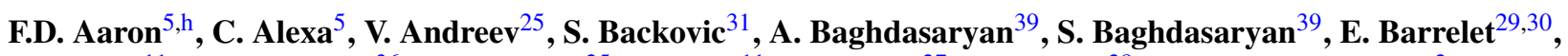
W. Bartel ${ }^{11}$, K. Begzsuren ${ }^{36}$, A. Belousov ${ }^{25}$, P. Belov ${ }^{11}$, J.C. Bizot ${ }^{27}$, V. Boudry ${ }^{28}$, I. Bozovic-Jelisavcic $^{2}$, J. Bracinik ${ }^{3}$, G. Brandt $^{11}$, M. Brinkmann ${ }^{11}$, V. Brisson ${ }^{27}$, D. Britzger ${ }^{11}$, D. Bruncko ${ }^{16}$, A. Bunyatyan ${ }^{13,39}$, G. Buschhorn $^{26, \dagger}$, L. Bystritskaya ${ }^{24}$, A.J. Campbell ${ }^{11}$, K.B. Cantun Avila ${ }^{22}$, F. Ceccopieri ${ }^{4}$, K. Cerny ${ }^{33}$, V. Cerny ${ }^{16, g}$, V. Chekelian ${ }^{26}$, J.G. Contreras ${ }^{22}$, J.A. Coughlan ${ }^{6}$, J. Cvach ${ }^{32}$, J.B. Dainton ${ }^{18}$, K. Daum ${ }^{38, a, c}$, B. Delcourt ${ }^{27}$, J. Delvax ${ }^{4}$, E.A. De Wolf ${ }^{4}$, C. Diaconu ${ }^{21}$, M. Dobre ${ }^{12, j, k}$, V. Dodonov ${ }^{13}$, A. Dossanov ${ }^{26}$, A. Dubak ${ }^{31, f}$, G. Eckerlin ${ }^{11}$, S. Egli ${ }^{37}$, A. Eliseev ${ }^{25}$, E. Elsen ${ }^{11}$, L. Favart ${ }^{4}$, A. Fedotov ${ }^{24}$, R. Felst ${ }^{11}$, J. Feltesse ${ }^{10}$, J. Ferencei ${ }^{16}$, D.-J. Fischer ${ }^{11}$, M. Fleischer ${ }^{11}$, A. Fomenko ${ }^{25}$, E. Gabathuler ${ }^{18}$, J. Gayler ${ }^{11}$, S. Ghazaryan ${ }^{11}$, A. Glazov ${ }^{11}$, L. Goerlich ${ }^{7}$, N. Gogitidze ${ }^{25}$, M. Gouzevitch ${ }^{11, e}$, C. Grab ${ }^{41}$, A. Grebenyuk ${ }^{11}$, T. Greenshaw ${ }^{18}$, B.R. Grell ${ }^{11}$, G. Grindhammer $^{26}$, S. Habib ${ }^{11}$, D. Haidt ${ }^{11}$, C. Helebrant ${ }^{11}$, R.C.W. Henderson ${ }^{17}$, E. Hennekemper ${ }^{15}$, H. Henschel ${ }^{40}$, M. Herbst $^{15}$, G. Herrera $^{23}$, M. Hildebrandt ${ }^{37}$, K.H. Hiller ${ }^{40}$, D. Hoffmann ${ }^{21}$, R. Horisberger ${ }^{37}$, T. Hreus ${ }^{4, d}$, F. Huber $^{14}$, M. Jacquet ${ }^{27}$, X. Janssen ${ }^{4}$, L. Jönsson ${ }^{20}$, H. Jung ${ }^{11,4,1}$, M. Kapichine ${ }^{9}$, I.R. Kenyon ${ }^{3}$, C. Kiesling ${ }^{26}$, M. Klein ${ }^{18}$, C. Kleinwort ${ }^{11}$, T. Kluge ${ }^{18}$, R. Kogler ${ }^{11}$, P. Kostka ${ }^{40}$, M. Kraemer ${ }^{11}$, J. Kretzschmar ${ }^{18}$, K. Krüger ${ }^{15}$, M.P.J. Landon ${ }^{19}$, W. Lange ${ }^{40}$, G. Laštovička-Medin ${ }^{31}$, P. Laycock ${ }^{18}$, A. Lebedev ${ }^{25}$, V. Lendermann ${ }^{15}$, S. Levonian ${ }^{11}$, K. Lipka ${ }^{11, j}$, B. List ${ }^{12}$, J. List ${ }^{11}$, R. Lopez-Fernandez ${ }^{23}$, V. Lubimov ${ }^{24}$, L. Lytkin ${ }^{9}$, A. Makankine ${ }^{9}$, E. Malinovski ${ }^{25}$, P. Marage $^{4}$, H.-U. Martyn ${ }^{1}$, S.J. Maxfield ${ }^{18}$, A. Mehta ${ }^{18}$, A.B. Meyer ${ }^{11}$, H. Meyer ${ }^{38}$, J. Meyer ${ }^{11}$, S. Mikocki ${ }^{7}$, I. Milcewicz-Mika ${ }^{7}$, F. Moreau ${ }^{28}$, A. Morozov $^{9}$, J.V. Morris ${ }^{6}$, M. Mudrinic ${ }^{2}$, K. Müller ${ }^{42}$, Th. Naumann $^{40}$, P.R. Newman ${ }^{3}$, C. Niebuhr ${ }^{11}$, D. Nikitin ${ }^{9}$, G. Nowak ${ }^{7}$, K. Nowak ${ }^{11}$, J.E. Olsson ${ }^{11}$, D. Ozerov ${ }^{24}$, P. Pahl ${ }^{11}$, V. Palichik ${ }^{9}$, I. Panagoulias ${ }^{11, b, x}$, M. Pandurovic ${ }^{2}$, Th. Papadopoulou ${ }^{11, b, x}$, C. Pascaud ${ }^{27}$, G.D. Patel $^{18}$, E. Perez $^{10, e}$, A. Petrukhin ${ }^{11}$, I. Picuric ${ }^{31}$, S. Piec ${ }^{11}$, H. Pirumov ${ }^{14}$, D. Pitzl ${ }^{11}$, R. Plačakyté ${ }^{12}$, B. Pokorny ${ }^{33}$, R. Polifka ${ }^{33}$, B. Povh ${ }^{13}$, V. Radescu $^{14}$, N. Raicevic ${ }^{31}$, T. Ravdandorj ${ }^{36}$, P. Reimer ${ }^{32}$, E. Rizvi ${ }^{19}$, P. Robmann ${ }^{42}$, R. Roosen ${ }^{4}$, A. Rostovtsev ${ }^{24}$, M. Rotaru ${ }^{5}$, J.E. Ruiz Tabasco ${ }^{22}$, S. Rusakov ${ }^{25}$, D. Š́lek ${ }^{33}$, D.P.C. Sankey ${ }^{6}$, M. Sauter ${ }^{14}$, E. Sauvan ${ }^{21}$, S. Schmitt ${ }^{11}$, L. Schoeffel ${ }^{10}$, A. Schöning ${ }^{14}$, H.-C. Schultz-Coulon ${ }^{15}$, F. Sefkow ${ }^{11}$, L.N. Shtarkov ${ }^{25}$, S. Shushkevich ${ }^{26}$, T. Sloan ${ }^{17}$,

I. Smiljanic $^{2}$, Y. Soloviev ${ }^{25}$, P. Sopicki ${ }^{7}$, D. South ${ }^{11}$, V. Spaskov ${ }^{9}$, A. Specka ${ }^{28}$, Z. Staykova ${ }^{4}$, M. Steder ${ }^{11}$, B. Stella ${ }^{34}$,

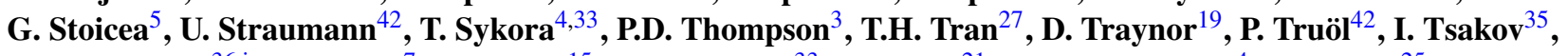
B. Tseepeldorj ${ }^{36, i}$, J. Turnau ${ }^{7}$, K. Urban ${ }^{15}$, A. Valkárová ${ }^{33}$, C. Vallée ${ }^{21}$, P. Van Mechelen ${ }^{4}$, Y. Vazdik ${ }^{25}$, D. Wegener $^{8}$, E. Wünsch ${ }^{11}$, J. Žáček ${ }^{33}$, J. Zálešák ${ }^{32}$, Z. Zhang ${ }^{27}$, A. Zhokin ${ }^{24}$, H. Zohrabyan ${ }^{39}$, F. Zomer ${ }^{27}$

\footnotetext{
${ }^{1}$ I. Physikalisches Institut der RWTH, Aachen, Germany

${ }^{2}$ Vinca Institute of Nuclear Sciences, University of Belgrade, 1100 Belgrade, Serbia

${ }^{3}$ School of Physics and Astronomy, University of Birmingham, Birmingham, $\mathrm{UK}^{\mathrm{n}}$

${ }^{4}$ Inter-University Institute for High Energies ULB-VUB, Brussels and Universiteit Antwerpen, Antwerpen, Belgium ${ }^{\circ}$

${ }^{5}$ National Institute for Physics and Nuclear Engineering (NIPNE), Bucharest, Romania ${ }^{\mathrm{y}}$

${ }^{6}$ Rutherford Appleton Laboratory, Chilton, Didcot, UK ${ }^{\mathrm{n}}$

${ }^{7}$ Institute for Nuclear Physics, Cracow, Poland ${ }^{\mathrm{P}}$

${ }^{8}$ Institut für Physik, TU Dortmund, Dortmund, Germany ${ }^{\mathrm{m}}$

${ }^{9}$ Joint Institute for Nuclear Research, Dubna, Russia

${ }^{10} \mathrm{CEA}$, DSM/Irfu, CE-Saclay, Gif-sur-Yvette, France

${ }^{11}$ DESY, Hamburg, Germany

${ }^{12}$ Institut für Experimentalphysik, Universität Hamburg, Hamburg, Germany ${ }^{\mathrm{m}}$

${ }^{13}$ Max-Planck-Institut für Kernphysik, Heidelberg, Germany

${ }^{14}$ Physikalisches Institut, Universität Heidelberg, Heidelberg, Germany ${ }^{\mathrm{m}}$

${ }^{15}$ Kirchhoff-Institut für Physik, Universität Heidelberg, Heidelberg, Germany ${ }^{\mathrm{m}}$

${ }^{16}$ Institute of Experimental Physics, Slovak Academy of Sciences, Košice, Slovak Republic ${ }^{\mathrm{r}}$

${ }^{17}$ Department of Physics, University of Lancaster, Lancaster, $\mathrm{UK}^{\mathrm{n}}$

${ }^{18}$ Department of Physics, University of Liverpool, Liverpool, $\mathrm{UK}^{\mathrm{n}}$

${ }^{19}$ Queen Mary and Westfield College, London, $\mathrm{UK}^{\mathrm{n}}$
} 
${ }^{20}$ Physics Department, University of Lund, Lund, Sweden ${ }^{\mathrm{s}}$

${ }^{21}$ CPPM, Aix-Marseille Univ., CNRS/IN2P3, 13288 Marseille, France

${ }^{22}$ Departamento de Fisica Aplicada, CINVESTAV, Mérida, Yucatán, Mexicov ${ }^{\mathrm{V}}$

${ }^{23}$ Departamento de Fisica, CINVESTAV IPN, México City, Mexicov ${ }^{v}$

${ }^{24}$ Institute for Theoretical and Experimental Physics, Moscow, Russia ${ }^{\mathrm{W}}$

${ }^{25}$ Lebedev Physical Institute, Moscow, Russia ${ }^{\mathrm{q}}$

${ }^{26}$ Max-Planck-Institut für Physik, München, Germany

${ }^{27}$ LAL, Université Paris-Sud, CNRS/IN2P3, Orsay, France

${ }^{28}$ LLR, Ecole Polytechnique, CNRS/IN2P3, Palaiseau, France

${ }^{29}$ LPNHE, Université Pierre et Marie Curie Paris 6, Paris, France

${ }^{30}$ Université Denis Diderot Paris 7, CNRS/IN2P3, Paris, France

${ }^{31}$ Faculty of Science, University of Montenegro, Podgorica, Montenegro ${ }^{\mathrm{z}}$

${ }^{32}$ Institute of Physics, Academy of Sciences of the Czech Republic, Praha, Czech Republic ${ }^{t}$

${ }^{33}$ Faculty of Mathematics and Physics, Charles University, Praha, Czech Republic ${ }^{\mathrm{t}}$

${ }_{35}^{34}$ Dipartimento di Fisica Università di Roma Tre and INFN Roma 3, Roma, Italy

${ }^{35}$ Institute for Nuclear Research and Nuclear Energy, Sofia, Bulgaria ${ }^{\mathrm{q}}$

${ }^{36}$ Institute of Physics and Technology of the Mongolian Academy of Sciences, Ulaanbaatar, Mongolia

${ }^{37}$ Paul Scherrer Institut, Villigen, Switzerland

${ }^{38}$ Fachbereich C, Universität Wuppertal, Wuppertal, Germany

${ }^{39}$ Yerevan Physics Institute, Yerevan, Armenia

${ }^{40}$ DESY, Zeuthen, Germany

${ }^{41}$ Institut für Teilchenphysik, ETH, Zürich, Switzerland ${ }^{\mathrm{u}}$

${ }^{42}$ Physik-Institut der Universität Zürich, Zürich, Switzerland ${ }^{u}$

Received: 29 June 2011 / Revised: 1 September 2011 / Published online: 13 October 2011

(C) The Author(s) 2011. This article is published with open access at Springerlink.com

Abstract The production of photons at very small angles with respect to the proton beam direction is studied in deepinelastic positron-proton scattering at HERA. The data are taken with the $\mathrm{H} 1$ detector in the years 2006 and 2007 and correspond to an integrated luminosity of $126 \mathrm{pb}^{-1}$. The analysis covers the range of negative four momentum transfer squared at the positron vertex $6<Q^{2}<100 \mathrm{GeV}^{2}$ and inelasticity $0.05<y<0.6$. Cross sections are measured for the most energetic photon with pseudorapidity $\eta>7.9$

a e-mail: daum@mail.desy.de

${ }^{\mathrm{b}}$ Also at Physics Department, National Technical University, Zografou Campus, GR-15773 Athens, Greece.

${ }^{c}$ Also at Rechenzentrum, Universität Wuppertal, Wuppertal, Germany.

${ }^{\mathrm{d}}$ Also at University of P.J. Šafárik, Košice, Slovak Republic.

eAlso at CERN, Geneva, Switzerland.

${ }^{\mathrm{f}}$ Also at Max-Planck-Institut für Physik, München, Germany.

${ }^{g}$ Also at Comenius University, Bratislava, Slovak Republic.

${ }^{h}$ Also at Faculty of Physics, University of Bucharest, Bucharest, Romania.

${ }^{\mathrm{i}}$ Also at Ulaanbaatar University, Ulaanbaatar, Mongolia.

${ }^{j}$ Supported by the Initiative and Networking Fund of the Helmholtz Association (HGF) under the contract VH-NG-401.

${ }^{\mathrm{k}}$ Absent on leave from NIPNE-HH, Bucharest, Romania.

${ }^{l}$ On leave of absence at CERN, Geneva, Switzerland.

${ }^{\mathrm{m}}$ Supported by the Bundesministerium für Bildung und Forschung, FRG, under contract numbers 05H09GUF, 05H09VHC, 05H09VHF, 05H16PEA.

${ }^{\mathrm{n}}$ Supported by the UK Science and Technology Facilities Council, and formerly by the UK Particle Physics and Astronomy Research Council. as a function of its transverse momentum $p_{T}^{\text {lead }}$ and longitudinal momentum fraction of the incoming proton $x_{L}^{\text {lead }}$. In addition, the cross sections are studied as a function of the sum of the longitudinal momentum fraction $x_{L}^{\text {sum }}$ of all photons in the pseudorapidity range $\eta>7.9$. The cross sections are normalised to the inclusive deep-inelastic scattering cross section and compared to the predictions of models of deep-inelastic scattering and models of the hadronic interactions of high energy cosmic rays.

${ }^{o}$ Supported by FNRS-FWO-Vlaanderen, IISN-IIKW and IWT and by Interuniversity Attraction Poles Programme, Belgian Science Policy.

pPartially Supported by Polish Ministry of Science and Higher Education, grant DPN/N168/DESY/2009.

${ }^{\mathrm{q}}$ Supported by the Deutsche Forschungsgemeinschaft.

${ }^{\mathrm{r}}$ Supported by VEGA SR grant No. 2/7062/27.

${ }^{\mathrm{s}}$ Supported by the Swedish Natural Science Research Council.

${ }^{\text {t}}$ Supported by the Ministry of Education of the Czech Republic under the projects LC527, INGO-LA09042 and MSM0021620859.

"Supported by the Swiss National Science Foundation.

${ }^{\mathrm{v}}$ Supported by CONACYT, México, grant 48778-F.

${ }^{\mathrm{w}}$ Russian Foundation for Basic Research (RFBR), grant No. 1329.2008.2 and Rosatom.

${ }^{\mathrm{x}}$ This project is co-funded by the European Social Fund (75\%) and National Resources (25\%) - (EPEAEK II) - PYTHAGORAS II.

${ }^{y}$ Supported by the Romanian National Authority for Scientific Research under the contract PN 09370101.

${ }^{\mathrm{z}}$ Partially Supported by Ministry of Science of Montenegro, No. 051/3-3352.

† Deceased. 


\section{Introduction}

Measurements of particle production at very small angles with respect to the proton beam direction (forward direction) in positron-proton collisions are important for the understanding of the fragmentation of the proton remnant. These measurements also provide important constraints for the modelling of the high energy air showers and thereby are very valuable for the understanding of high energy cosmic ray data $[1,2]$. The $\mathrm{H} 1$ and ZEUS experiments at the $e^{ \pm} p$ collider HERA have published several analyses on the production of forward protons and neutrons which carry a large fraction of the longitudinal momentum of the incoming proton [3-7]. These measurements probe different mechanisms related to the baryon production in forward direction, such as elastic scattering of the proton, diffractive dissociation, pion exchange and string fragmentation. In particular, these measurements test the hypothesis of limiting fragmentation $[8,9]$, according to which, in the high-energy limit, the cross section for the inclusive production of particles in the target fragmentation region is independent of the incident projectile energy. This hypothesis implies, that in deepinelastic scattering (DIS) forward particle production cross sections are independent of the Bjorken- $x$ and the virtuality of the exchanged photon $Q^{2}$.

The measurement of the photon production in the forward direction can provide new input to the understanding of proton fragmentation, and is complementary to forward baryon measurements. The production of photons and $\pi^{0}$ mesons in the proton fragmentation region has been studied in $\bar{p} p$ and $p p$ collisions at SPS and the LHC colliders [10, 11]. The analysis presented here is the first measurement of very forward photons in DIS $e^{+} p$ collisions at HERA. The photons are detected at very small angles below $0.75 \mathrm{mrad}$ with respect to proton beam direction. It relies on the upgraded H1 Forward Neutron Calorimeter (FNC) which includes an electromagnetic section.

\section{Experimental procedure and data analysis}

The data used in this analysis were collected with the H1 detector at HERA in the years 2006 and 2007 and correspond to an integrated luminosity of $126 \mathrm{pb}^{-1}$. During the period corresponding to the analysis data set HERA collided positrons and protons with energies of $E_{e}=27.6 \mathrm{GeV}$ and $E_{p}=920 \mathrm{GeV}$, respectively, corresponding to a centre-ofmass energy of $\sqrt{s}=319 \mathrm{GeV}$.

\section{$2.1 \mathrm{H} 1$ detector}

A detailed description of the $\mathrm{H} 1$ detector can be found elsewhere $[12-16,20]$. Only the detector components relevant to this analysis are briefly described here. The origin of the right-handed $\mathrm{H} 1$ coordinate system is the nominal $e^{+} p$ interaction point. The direction of the proton beam defines the positive $z$ axis; the polar angle $\theta$ is measured with respect to this axis. Transverse momenta are measured in the $x-y$ plane. The pseudorapidity is defined by $\eta=-\ln \left(\tan \frac{\theta}{2}\right)$ and is measured in the lab frame. The polar angles $\theta<$ $0.75 \mathrm{mrad}$ correspond to pseudorapidity range $\eta>7.9$.

The interaction region is surrounded by a two-layer silicon strip detector and two large concentric drift chambers. Charged particle momenta are measured in the angular range $25^{\circ}<\theta<155^{\circ}$. The tracking system is surrounded by a finely segmented Liquid Argon (LAr) calorimeter, which covers the polar angle range of $4^{\circ}<\theta<154^{\circ}$ with full azimuthal acceptance. The LAr calorimeter consists of an electromagnetic section with lead absorber and a hadronic section with steel absorber. The total depth of the LAr calorimeter ranges from 4.5 to 8 hadronic interaction lengths. The backward region $\left(153^{\circ}<\theta<177.8^{\circ}\right)$ is covered by a lead/scintillating-fibre calorimeter ( $\mathrm{SpaCal})$. Its main purpose is the detection of the scattered positron. The energy resolution for positrons is $\sigma(E) / E \approx 7.1 \% / \sqrt{E[\mathrm{GeV}]} \oplus$ $1 \%$, as determined in test beam measurements [16]. The LAr and SpaCal calorimeters are surrounded by a superconducting solenoid which provides a uniform magnetic field of $1.16 \mathrm{~T}$ along the beam direction.

The luminosity is measured via the Bethe-Heitler Bremsstrahlung process $e p \rightarrow e^{\prime} p \gamma$, the final state photon being detected in a tungsten/quartz-fibre sampling calorimeter at $z=-103 \mathrm{~m}$.

The data sample of this analysis was collected using triggers which require the scattered positron to be measured in the SpaCal. The trigger efficiency is about $96 \%$ for the analysis phase space as determined from data using independently triggered data.

\subsection{Detection of forward neutral particles}

Neutral particles produced at very small polar angles can be detected in the FNC calorimeter, which is situated at a polar angle of $0^{\circ}$, at $z=+106 \mathrm{~m}$ from the interaction point. A schematic view of the H1 FNC used during the HERA-II running period is shown in Fig. 1a. A detailed description of the detector is given in [7]. The FNC consists of the Main Calorimeter and the Preshower Calorimeter. The Main Calorimeter is a lead-scintillator sandwich calorimeter with a total length of 8.9 nuclear interaction lengths. The Preshower Calorimeter is a $40 \mathrm{~cm}$ long leadscintillator sandwich calorimeter. The length corresponds to about 60 radiation lengths. The Preshower Calorimeter is composed of 24 planes: the first 12 planes each consist of a lead plate of $7.5 \mathrm{~mm}$ thickness and a scintillator plate of $2.6 \mathrm{~mm}$ thickness. The second 12 planes each consist of a lead plate of $14 \mathrm{~mm}$ thickness and a scintillator 
Fig. 1 (a) A schematic view of the H1 FNC. (b) Layout of 9 vertical and 9 horizontal readout strips of the Preshower Calorimeter. The hatched area shows the geometrical acceptance window defined by the beam-line elements. The area corresponding to $\eta>7.9$ is indicated by the dashed circle (a)

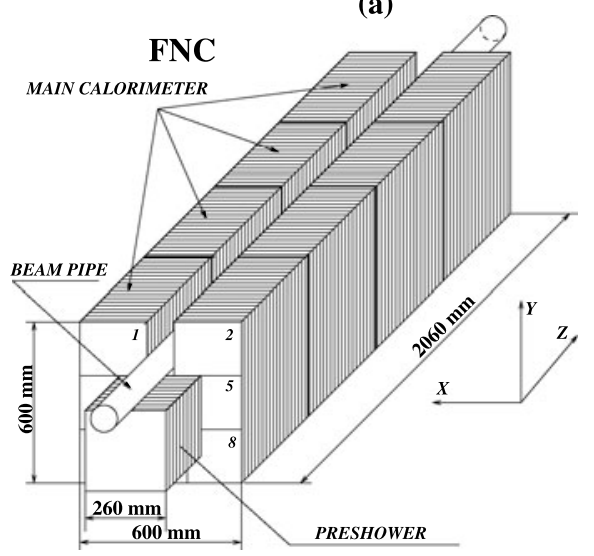

(b)

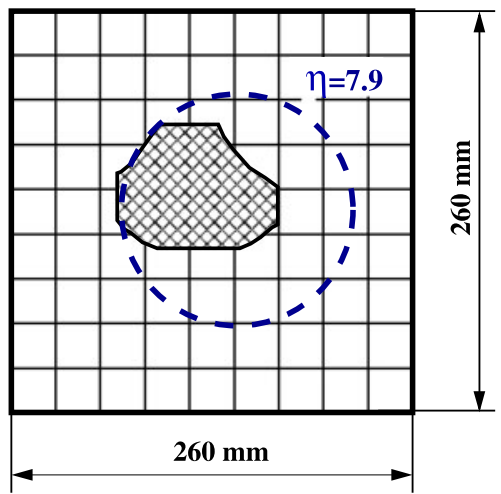

plate of $5.2 \mathrm{~mm}$ thickness. The transverse size of the scintillating plates is $26 \times 26 \mathrm{~cm}^{2}$. Each scintillating plate has 45 grooves with $1.2 \mathrm{~mm}$ wavelength shifter fibres attached down one side. The orientation of fibres alternates from horizontal to vertical in consecutive planes. For each plane, the fibres are bundled into nine strips of five fibres each. Longitudinally, all strips are combined leading to 9 vertical and 9 horizontal towers which are finally connected to 18 photomultipliers.

The acceptance of the FNC is defined by the aperture of the HERA beam-line magnets and is limited to scattering angles of $\theta \lesssim 0.8 \mathrm{mrad}$ with approximately $30 \%$ azimuthal coverage. The geometrical acceptance window of the FNC is shown in Fig. 1b together with the layout of the Preshower Calorimeter readout strips.

The longitudinal segmentation of the FNC allows efficient discrimination of photons from hadrons. The photon reconstruction algorithm is based on the fact that electromagnetic showers are fully contained in the Preshower Calorimeter with no energy deposits above the noise level in the Main Calorimeter. For high energy neutrons most of the energy is contained in the Main Calorimeter. However, low energy neutrons deposit large fractions of their energy in the Preshower Calorimeter. The fraction of neutrons which can be misidentified as photons is about $10 \%$ for $90 \mathrm{GeV}$ neutrons decreasing to below $1 \%$ for neutrons with an energy of $200 \mathrm{GeV}$, as determined from the Monte Carlo (MC) simulation. The energy deposits in the FNC which are contained in the Preshower Calorimeter are classified as electromagnetic clusters and are considered as photon candidates. The detection and reconstruction efficiency for photons in the measured angular range $\theta<0.75 \mathrm{mrad}$, as estimated from MC simulation, is about $85 \%$ for $100 \mathrm{GeV}$ photons increasing to $95 \%$ for photons with energies of $900 \mathrm{GeV}$. Losses are mainly due to interactions with the beampipe.

All modules of the FNC were initially calibrated at CERN using 120-230 GeV electron and 120-350 GeV hadron beams. After the calorimeter was installed at DESY, the stability of calibration constants was monitored using interactions between the proton beam and residual gas in the beam pipe, as described in [7]. Refined calibration constants for electromagnetic showers are determined using an iterative procedure based on the assumption that the maximum photon energy, $E_{\gamma}^{\max }$, as measured in the Preshower Calorimeter, is expected to be equal to the proton beam energy in case of unlimited statistics. This calibration procedure also utilises data from HERA runs with reduced proton beam energies of $460 \mathrm{GeV}$ and $575 \mathrm{GeV}$. The validity of this algorithm is tested with MC simulation.

The measured photon energy spectra for the three proton beam energies are displayed in Fig. 2a. The correlation between the beam energy and the maximum photon energy $E_{\gamma}^{\max }$ as determined by the iterative procedure and after applying the calibration is shown in Fig. 2b. Using this calibration procedure, the linearity of the energy response and the absolute energy scale are verified to a precision of $5 \%$.

The energy resolution of the FNC calorimeter for electromagnetic showers is $\sigma(E) / E \approx 20 \% / \sqrt{E[\mathrm{GeV}]} \oplus 2 \%$, as determined in test beam measurements. The spatial resolution for single electromagnetic showers and for those hadronic showers which started to develop in the Preshower Calorimeter is about $2 \mathrm{~mm}$.

\subsection{Kinematics and event selection}

The kinematic variables used to describe high energy DIS interactions are the exchanged photon virtuality $Q^{2}$, the inelasticity $y$ and the Bjorken scaling variable $x_{B j}$. They are defined as

$Q^{2}=-q^{2}, \quad x_{B j}=\frac{Q^{2}}{2 p \cdot q}, \quad y=\frac{p \cdot q}{p \cdot k}$,

where $p, k$ and $q$ are the four-momenta of the incident proton, the incident positron and the virtual photon, respectively. 
Fig. 2 (a) The measured photon energy spectra for three proton beam energies. (b) The correlation between the proton beam energy and $E_{\gamma}^{\max }$

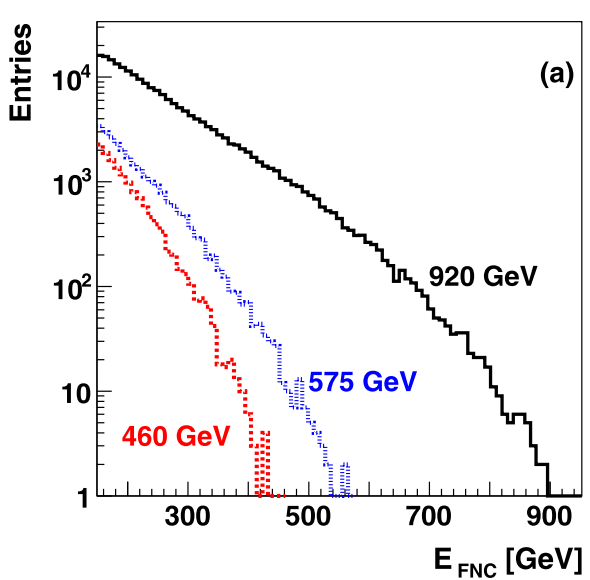

The selection of DIS events is based on the identification of the scattered positron as the most energetic compact calorimetric deposit in the SpaCal with an energy $E_{e}^{\prime}>11 \mathrm{GeV}$ and a polar angle $156^{\circ}<\theta_{e}^{\prime}<175^{\circ}$. The $z$ coordinate of the primary event vertex is required to be within $\pm 35 \mathrm{~cm}$ of the nominal position of the interaction point. The hadronic final state is reconstructed using an energy flow algorithm which combines charged particles measured in the tracker with information from the SpaCal and LAr calorimeters [18, 19]. To suppress events with hard initial state radiation, as well as events originating from non$e^{+} p$ interactions, the quantity $\sum E-p_{z}$, summed over all reconstructed final state particles including the positron, is required to lie between $35 \mathrm{GeV}$ and $70 \mathrm{GeV}$. This quantity, which uses the energy and longitudinal momentum component of each final state particle, is expected to be twice the electron beam energy for fully contained events. In addition, events are restricted to the kinematic range $6<Q^{2}<$ $100 \mathrm{GeV}^{2}$ and $0.05<y<0.6$. These variables are reconstructed using a technique which optimises the resolution throughout the measured $y$ range, exploiting information from both the scattered positron and the hadronic final state [17]. The data sample of the DIS events contains about 9.2 million events.

Events containing forward photons are selected by requiring an electromagnetic cluster in the FNC with a pseudorapidity above 7.9 and an energy above $92 \mathrm{GeV}$, which corresponds to the longitudinal momentum fraction $x_{L}=$ $E_{\gamma} / E_{p}>0.1$, where $E_{p}$ and $E_{\gamma}$ are the proton beam and forward photon energy, respectively. The data sample contains 78740 events.

In this analysis normalised differential cross sections are measured for the most energetic forward photon (leading photon) with pseudorapidity $\eta>7.9$ as a function of its longitudinal momentum fraction $x_{L}^{\text {lead }}$ and transverse momentum $p_{T}^{\text {lead }}$, in the range $0.1<x_{L}^{\text {lead }}<0.7$. Cross sections are also measured as a function of the sum of longitudinal momentum fractions of all forward photons with $\eta>7.9$,
$x_{L}^{\text {sum }}=\sum x_{L}$, in the range $0.1<x_{L}^{\text {sum }}<0.95$. These cross sections are given as the fraction of DIS events having forward photon in the $\eta-x_{L}$ regions given above. Finally, the ratio of the forward photon production cross section to the inclusive DIS cross section is presented as a function of $Q^{2}$ and $x_{B j}$.

\subsection{Monte Carlo simulations and corrections to the data}

Monte Carlo simulations are used to correct the data for the effects of detector acceptance, inefficiencies, migrations between measurement bins due to finite detector resolution and QED radiation from the positron. All generated events are passed through a GEANT3 [21] based simulation of the H1 apparatus and are then processed using the same reconstruction and analysis chain as is used for the data.

The DJANGOH [22] program is used to generate inclusive DIS events. It is based on leading order electroweak cross sections and takes into account QCD effects up to order $\alpha_{s}$. Higher order QCD effects are simulated using leading log parton showers as implemented in LEPTO [23], or using the Colour Dipole Model (CDM) as implemented in ARIADNE [24]. Subsequent hadronisation effects are modelled using the Lund string fragmentation model as implemented in JETSET [25, 26]. Higher order electroweak processes are simulated using an interface to HERACLES [27]. The LEPTO program includes the simulation of soft colour interactions (SCI) [28], in which the production of diffraction-like configurations is enhanced via non-perturbative colour rearrangements between the outgoing partons. In the measured $x_{L}$ range, omitting the SCI in the LEPTO would decrease the predicted yield of forward photons by $5 \%$ at lowest $x_{L}$ and $2 \%$ at highest $x_{L}$. The simulation with $\mathrm{CDM}$ uses the parameters tuned to describe $\mathrm{H} 1$ forward jet measurements [29]. The DJANGOH MC simulations are calculated using the H1PDF 2009 parameterisation [30] of the parton distributions in the proton. In the following, the predictions based on LEPTO and ARIADNE are denoted LEPTO and CDM, respectively. 
The measurements are also compared with the predictions of several hadronic interaction models which are commonly used for the simulation of cosmic ray air shower cascades: EPOS [31], QGSJET 01 [32, 33], QGSJET II-03 [34, 35] and SIBYLL [36, 39]. These models are based on Regge theory [38], the Gribov's Reggeon calculus [37], and on perturbative QCD. They use an unitarisation procedure to reconstruct amplitudes for exclusive processes and to determine the total and elastic cross sections. Central elements of these models are the production of mini-jets and the formation of colour strings that fragment into hadrons. Whereas the Regge-Gribov approximation is applied to hadrons as interacting objects in the case of QGSJET and SIBYLL, it is extended to include partonic constituents in EPOS. Furthermore the models differ in the treatment of saturation effects at high parton densities at small $x$ and in the treatment of the hadronic remnants in collisions. The programs are interfaced with the PHOJET program [40] for the simulation of $e^{+} p$ interactions.

In all of these models, the main source of forward photons is the decay of $\pi^{0}$ mesons produced from the hadronisation of the proton remnant. The measured distributions may contain background arising from several sources. The background from photoproduction processes, where the positron is scattered into the backward beam-pipe and a particle from the hadronic final state fakes the positron signature in the SpaCal, is estimated using the PHOJET MC generator and found to be negligible. The selected sample may contain background from neutrons reconstructed as electromagnetic clusters as explained above. For cluster energies above $92 \mathrm{GeV}$ this background is found to be negligible according to the MC simulation. The background from the random coincidences of DIS events with a beam-related background signals in the FNC is estimated by combining DIS events with forward particles in adjacent bunch-crossings. It is found to be smaller than $1 \%$. The background contributions are not subtracted from the measured cross sections.

Two or more particles entering the FNC are reconstructed as a single cluster due to the relatively large size of the FNC readout modules in combination with a small geometrical acceptance window. According to the MC simulation, low energetic clusters reconstructed in the FNC mainly originate from single photons. The contribution from two photons increases almost linearly from $10 \%$ at about $450 \mathrm{GeV}$ to $80 \%$ at $900 \mathrm{GeV}$ (the contribution from three and more photons is below 1\%). Therefore, the measurement of the cross section of single photon production is limited to $x_{L}<0.7$, while the measurement of the total forward photon production cross section is extended to larger $x_{L}$.

Factors determined from MC are used to correct distributions at the level of reconstructed particles back to the hadron level on a bin-by-bin basis. These correction factors include the effects of QED radiation from the positron. For the calculation of the correction factors the simulations are reweighted to describe the $x_{L}$ distributions of the data. The average of the correction factors determined from LEPTO and CDM is used. The size of the correction factors varies between 2 and 3.5 for $x_{L}^{\text {lead }}$, between 3 and 4 for $x_{L}^{\text {sum }}$, between 2.5 and 12 for $p_{T}^{\text {lead }}$ and are about 3.2 for the $Q^{2}$ and $x_{B j}$ distributions. They are dominated by the non-uniform azimuthal acceptance of the FNC, which is about $30 \%$ on average. The bin purities, defined as the fraction of events reconstructed in a particular bin that originate from that bin on hadron level, vary between $75 \%$ and $95 \%$.

\subsection{Systematic uncertainties}

The systematic uncertainties on the cross section measurements are determined using MC simulations, by propagating the corresponding uncertainty sources through the full analysis chain.

As the cross sections are normalised to the inclusive DIS cross section measured in this analysis, some important systematic uncertainties, such as the trigger efficiency, the luminosity and the uncertainties related to the reconstruction of the scattered positron and of the hadronic final state are largely reduced or cancel. Uncertainties on the measurements of the scattered positron energy (1\%) and angle $(1 \mathrm{mrad})$, the energy of the hadronic final state $(4 \%)$, and the uncertainty on the trigger efficiency $(1 \%)$ lead to an average combined uncertainty of up to $2 \%$.

The absolute electromagnetic energy scale of the FNC is known to a precision of 5\% as described in Sect. 2.2. This leads to an uncertainty of $1 \%$ on the cross section measurement at low energies, increasing to $35 \%$ for the largest $x_{L}$ values. The acceptance of the FNC calorimeter is defined by the interaction point and the geometry of the HERA magnets and is determined using MC simulations. The uncertainty of the impact position of the photon on the FNC is due to beam inclination and the uncertainty on the FNC position. It is estimated to be $5 \mathrm{~mm}$. This results in uncertainties on the FNC acceptance determination of up to $15 \%$ for the $x_{L}$ distributions and up to $60 \%$ for the $p_{T}^{\text {lead }}$ distribution. These effects are strongly correlated between measurement bins. For the $Q^{2}$ and $x_{B j}$ measurements, these effects lead to normalisation uncertainty of approximately $7 \%$.

The systematic uncertainty arising from the model dependence of the data correction is taken as the difference of the corrections calculated using the LEPTO and CDM models. The resulting uncertainty on the cross-section increases from $1 \%$ to $6 \%$ for the $x_{L}^{\text {lead }}$ and $p_{T}^{\text {lead }}$ distributions, from $2 \%$ to $20 \%$ for the $x_{L}^{\text {sum }}$ distribution, and from $1 \%$ to $2 \%$ for the $Q^{2}$ and $x_{B j}$ distributions. Using different parton distribution functions in the $\mathrm{MC}$ simulation results in a negligible change in the cross section.

The systematic errors shown in the figures and table are calculated as the quadratic sum of all contributions, which 
may vary from point to point. The total systematic error for the normalised cross section measurements ranges between $8 \%$ and $18 \%$ for $x_{L}^{\text {lead }}, 6 \%$ and $58 \%$ for $p_{T}^{\text {lead }}, 8 \%$ and $44 \%$ for $x_{L}^{\text {sum }}$ and $7 \%$ and $8 \%$ for $Q^{2}$ and $x_{B j}$.

\section{Results}

The measured normalised differential cross sections for the production of very forward photons in the pseudorapidity range $\eta>7.9$ in DIS in the kinematic range $6<Q^{2}<$ $100 \mathrm{GeV}^{2}$ and $0.05<y<0.6$, are presented in Table 1 and Figs. 3, 4 and 5. The measurements are presented in Figs. 3 and 4 as a function of $x_{L}^{\text {lead }}$ and $p_{T}^{\text {lead }}$ of the most energetic photon with $0.1<x_{L}^{\text {lead }}<0.7$. The results as a function of the sum of longitudinal momentum fractions $x_{L}^{\text {sum }}$ of all photons with $\eta>7.9$ are presented in Fig. 5.

The data are compared with the predictions of models for inclusive DIS (LEPTO and CDM) and models of hadronic interactions (EPOS, SIBYLL and two versions of QGSJET). The ratios of MC model predictions to the measurements are shown separately.

Table 1 Normalised cross sections of forward photon production with $\eta>7.9$ in DIS in the kinematic region $6<Q^{2}<100 \mathrm{GeV}^{2}$ and $0.05<y<0.6$ as a function of $x_{L}^{\text {lead }}$ and $p_{T}^{\text {lead }}$ of the most energetic photon in the energy range $0.1<x_{L}^{\text {lead }}<0.7$ and as a function $x_{L}^{\text {sum }}$.
All models tested in this paper overestimate the total rate of forward photons. The LEPTO and CDM models predict about $70 \%$ more photons than measured, while EPOS, SIBYLL and QGSJET overestimate the rate of photons by about $30 \%$ to $50 \%$. In contrast to the excess of photons in the CDM, the same model predicts a too low rate of forward neutrons as observed in previous $\mathrm{H} 1$ analysis of forward neutron production [7].

The shapes of all measured distributions are well described by LEPTO. The CDM predicts harder $x_{L}$ and $p_{T}$ spectra. The QGSJET model overestimates the measured cross sections by about $40 \%$ at lowest $x_{L}$ and $p_{T}$ but is consistent with the data within the experimental uncertainties elsewhere. The EPOS and SIBYLL models predict harder $x_{L}$ spectra, but describe reasonably the shape of $p_{T}$ distribution.

A measurement of the energy spectra of single photons produced in $p p$ collisions at $7 \mathrm{TeV}$ centre-of-mass energy at the LHC has been recently reported by the LHCf Collaboration [11] for the pseudorapidity ranges $8.81<$ $\eta<8.99$ and $\eta>10.94$. Due to the different kinematic ranges of the two measurements a direct comparison of the H1 and LHCf results is not possible. The LHCf mea-

For each measurement, the statistical, the total systematic, the uncorrelated systematic uncertainties, and the bin-to-bin correlated systematic uncertainties due to the FNC absolute energy scale, the impact position of the FNC and the model dependence of data correction are given

\begin{tabular}{|c|c|c|c|c|c|c|c|}
\hline \multirow[b]{2}{*}{$x_{L}^{\text {lead }}$ range } & \multirow[b]{2}{*}{$\frac{1}{\sigma_{\mathrm{DIS}}} \frac{d \sigma}{d x_{L}^{\text {lead }}}$} & \multirow[b]{2}{*}{$\delta_{\text {stat. }}$} & \multirow[b]{2}{*}{$\delta_{\text {total sys. }}$} & \multirow[b]{2}{*}{$\delta_{\text {uncorrel.sys. }}$} & \multicolumn{3}{|c|}{ Correlated sys. uncertainty } \\
\hline & & & & & $\delta_{E_{\mathrm{FNC}}}$ & $\delta_{X Y_{\mathrm{FNC}}}$ & $\delta_{\text {model }}$ \\
\hline $0.10 \div 0.22$ & 0.134 & 0.001 & 0.011 & 0.002 & 0.001 & 0.011 & 0.001 \\
\hline $0.22 \div 0.34$ & 0.0577 & 0.0005 & 0.0061 & 0.0012 & 0.0029 & 0.0052 & 0.0008 \\
\hline $0.34 \div 0.46$ & 0.0226 & 0.0003 & 0.0029 & 0.0005 & 0.0018 & 0.0023 & 0.0003 \\
\hline $0.46 \div 0.58$ & 0.00764 & 0.00017 & 0.00123 & 0.00029 & 0.00061 & 0.00099 & 0.00027 \\
\hline $0.58 \div 0.70$ & 0.00229 & 0.00008 & 0.00048 & 0.00017 & 0.00025 & 0.00034 & 0.00016 \\
\hline \multirow[b]{2}{*}{$\begin{array}{l}p_{T}^{\text {lead }} \text { range } \\
{[\mathrm{GeV}]}\end{array}$} & \multirow[b]{2}{*}{$\begin{array}{l}\frac{1}{\sigma_{\mathrm{DIS}}} \frac{d \sigma}{d p_{T}^{\text {lead }}} \\
{\left[\mathrm{GeV}^{-1}\right]}\end{array}$} & \multirow[b]{2}{*}{$\begin{array}{l}\delta_{\text {stat. }} \\
{\left[\mathrm{GeV}^{-1}\right]}\end{array}$} & \multirow[b]{2}{*}{$\begin{array}{l}\delta_{\text {total sys. }} \\
{\left[\mathrm{GeV}^{-1}\right]}\end{array}$} & \multirow[b]{2}{*}{$\begin{array}{l}\delta_{\text {uncorrel.sys. }} \\
{\left[\mathrm{GeV}^{-1}\right]}\end{array}$} & \multicolumn{3}{|c|}{ Correlated sys. uncertainty } \\
\hline & & & & & $\begin{array}{l}\delta_{E_{\mathrm{FNC}}} \\
{\left[\mathrm{GeV}^{-1}\right]}\end{array}$ & $\begin{array}{l}\delta_{X Y_{\mathrm{FNC}}} \\
{\left[\mathrm{GeV}^{-1}\right]}\end{array}$ & $\begin{array}{l}\delta_{\text {model }} \\
{\left[\mathrm{GeV}^{-1}\right]}\end{array}$ \\
\hline $0.0 \div 0.1$ & 0.159 & 0.001 & 0.010 & 0.003 & 0.005 & 0.008 & 0.001 \\
\hline $0.1 \div 0.2$ & 0.0971 & 0.0010 & 0.0116 & 0.0041 & 0.0068 & 0.0078 & 0.0034 \\
\hline $0.2 \div 0.3$ & 0.0220 & 0.0005 & 0.0056 & 0.0010 & 0.0024 & 0.0048 & 0.0008 \\
\hline \multirow[t]{2}{*}{$0.3 \div 0.4$} & 0.00395 & 0.00029 & 0.00229 & 0.00025 & 0.00087 & 0.00209 & 0.00020 \\
\hline & & & & & \multicolumn{3}{|c|}{ Correlated sys. uncertainty } \\
\hline$x_{L}^{\text {sum }}$ range & $\frac{1}{\sigma_{\mathrm{DIS}}} \frac{d \sigma}{d x_{L}^{\mathrm{sum}}}$ & $\delta_{\text {stat. }}$ & $\delta_{\text {total sys. }}$ & $\delta_{\text {uncorrel.sys. }}$ & $\delta_{E_{\mathrm{FNC}}}$ & $\delta_{X Y_{\mathrm{FNC}}}$ & $\delta_{\text {model }}$ \\
\hline $0.10 \div 0.27$ & 0.110 & 0.001 & 0.009 & 0.002 & 0.001 & 0.009 & 0.002 \\
\hline $0.27 \div 0.44$ & 0.0353 & 0.0003 & 0.0038 & 0.0009 & 0.0018 & 0.0032 & 0.0007 \\
\hline $0.44 \div 0.61$ & 0.0115 & 0.00021 & 0.0018 & 0.0007 & 0.0009 & 0.0012 & 0.0006 \\
\hline $0.61 \div 0.78$ & 0.00315 & 0.00011 & 0.00068 & 0.00032 & 0.00032 & 0.00041 & 0.00031 \\
\hline $0.78 \div 0.95$ & 0.000468 & 0.000039 & 0.000172 & 0.000050 & 0.000140 & 0.000070 & 0.000050 \\
\hline
\end{tabular}


Fig. 3 Normalised cross sections of forward photon production in DIS as a function of $x_{L}^{\text {lead }}$ in the region $\eta>7.9$,

$0.1<x_{L}^{\text {lead }}<0.7$,

$6<Q^{2}<100 \mathrm{GeV}^{2}$ and

$0.05<y<0.6$. The data are

compared to two predictions of the DJANGOH Monte Carlo simulation, using LEPTO and $\mathrm{CDM}$ to simulate higher orders. Also shown are models of hadronic interactions, QGSJET, EPOS and SIBYLL. The lower row shows the ratios of the Monte Carlo predictions to the data. The error bars show the total experimental uncertainty, defined as the quadratic sum of the statistical and systematic uncertainties

Fig. 4 Normalised cross sections of forward photon production in DIS as a function of $p_{T}^{\text {lead }}$ in the region $\eta>7.9$,

$0.1<x_{L}^{\text {lead }}<0.7$,

$6<Q^{2}<100 \mathrm{GeV}^{2}$ and

$0.05<y<0.6$. The data are compared to two predictions of the DJANGOH Monte Carlo simulation, using LEPTO and CDM to simulate higher orders. Also shown are models of hadronic interactions, QGSJET, EPOS and SIBYLL. The lower row shows the ratios of the Monte Carlo predictions to the data. The error bars show the total experimental uncertainty, defined as the quadratic sum of the statistical and systematic uncertainties
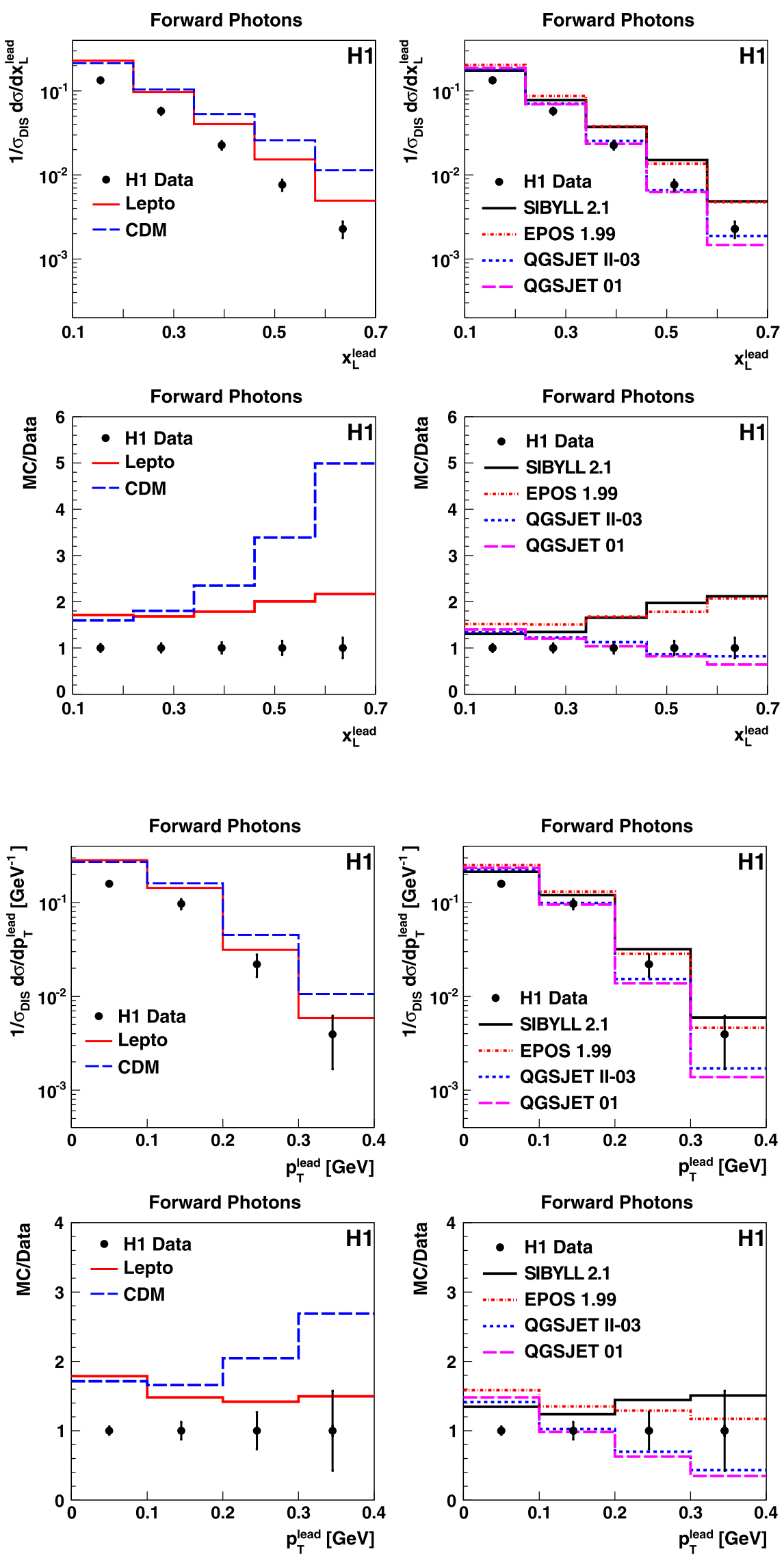
Fig. 5 Normalised cross sections of forward photon production in DIS as a function of $x_{L}^{\text {sum }}$ in the region $\eta>7.9$, $6<Q^{2}<100 \mathrm{GeV}^{2}$ and $0.05<y<0.6$. The data are compared to two predictions of the DJANGOH Monte Carlo simulation, using LEPTO and CDM to simulate higher orders. Also shown are models of hadronic interactions, QGSJET, EPOS and SIBYLL. The lower row shows the ratios of the Monte Carlo predictions to the data. The error bars show the total experimental uncertainty, defined as the quadratic sum of the statistical and systematic uncertainties

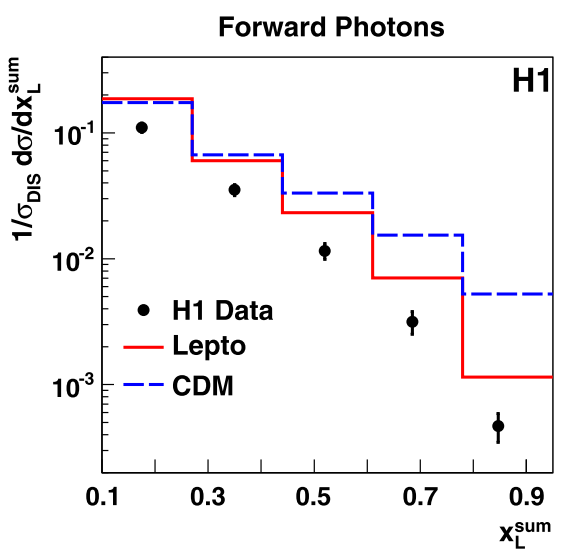

Forward Photons

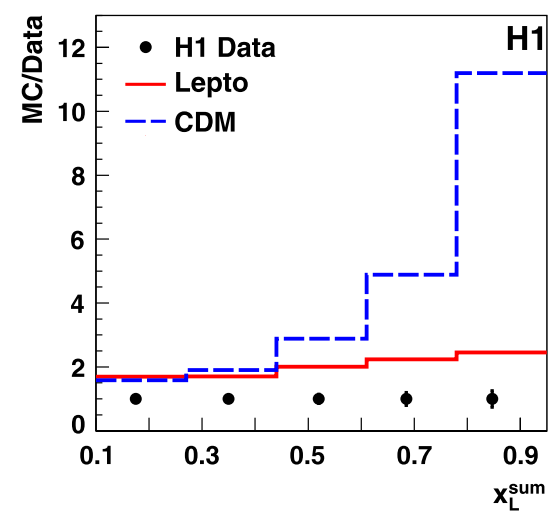

Forward Photons

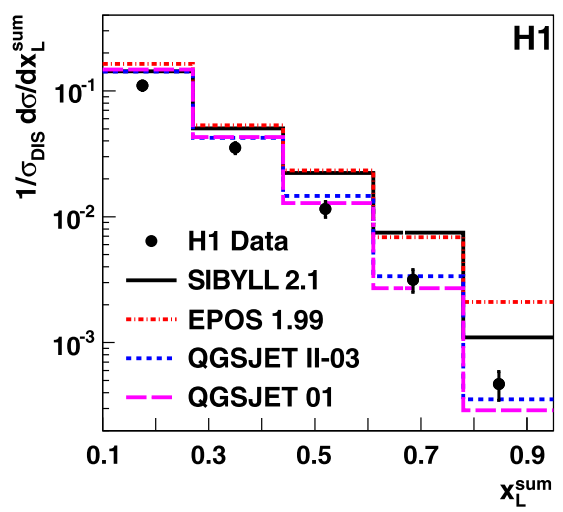

Forward Photons

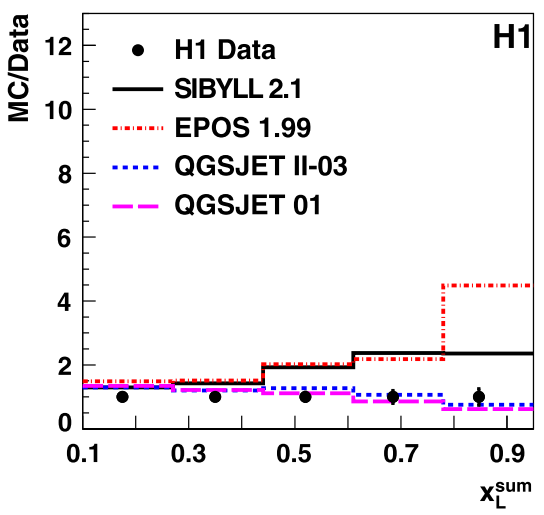

Table 2 Fraction of DIS events with forward photons in the kinematic region $6<Q^{2}<100 \mathrm{GeV}^{2}$ and $0.05<y<0.6$ and the pseudorapidity of the photon $\eta>7.9$. For each measurement, the statistical, the total systematic, the uncorrelated systematic uncertainties, and the bin-to- bin correlated systematic uncertainties due to the FNC absolute energy scale, the impact position of the FNC and the model dependence of data correction are given

\begin{tabular}{|c|c|c|c|c|c|c|c|}
\hline \multirow[b]{2}{*}{$Q^{2}$ range $\left[\mathrm{GeV}^{2}\right]$} & \multirow[b]{2}{*}{$\frac{\sigma_{\mathrm{DIS}}^{\gamma}\left(Q^{2}\right)}{\sigma_{\mathrm{DIS}}\left(Q^{2}\right)}$} & \multirow[b]{2}{*}{$\delta_{\text {stat. }}$} & \multirow[b]{2}{*}{$\delta_{\text {total sys. }}$} & \multirow[b]{2}{*}{$\delta_{\text {uncorrel.sys. }}$} & \multicolumn{3}{|c|}{ Correlated sys. uncertainty } \\
\hline & & & & & $\delta_{E_{\mathrm{FNC}}}$ & $\delta_{X Y_{\mathrm{FNC}}}$ & $\delta_{\text {model }}$ \\
\hline $6.0 \div 24.8$ & 0.0276 & 0.0001 & 0.0020 & 0.0003 & 0.0011 & 0.0017 & 0.0001 \\
\hline $24.8 \div 43.6$ & 0.0265 & 0.0003 & 0.0020 & 0.0003 & 0.0011 & 0.0016 & 0.0001 \\
\hline $43.6 \div 62.4$ & 0.0265 & 0.0005 & 0.0020 & 0.0004 & 0.0011 & 0.0016 & 0.0001 \\
\hline $62.4 \div 81.2$ & 0.0261 & 0.0007 & 0.0020 & 0.0005 & 0.0010 & 0.0016 & 0.0001 \\
\hline \multirow[t]{2}{*}{$81.2 \div 100.0$} & 0.0279 & 0.0011 & 0.0021 & 0.0005 & 0.0011 & 0.0017 & 0.0001 \\
\hline & & & & & \multicolumn{3}{|c|}{ Correlated sys. uncertainty } \\
\hline$x_{B j}$ range & $\frac{\sigma_{\mathrm{DIS}}^{\gamma}\left(x_{B j}\right)}{\sigma_{\mathrm{DIS}}\left(x_{B j}\right)}$ & $\delta_{\text {stat. }}$ & $\delta_{\text {total sys. }}$ & $\delta_{\text {uncorrel.sys. }}$ & $\overline{\delta_{E}}$ & $\delta_{\mathrm{XY}}$ & $\delta_{\text {model }}$ \\
\hline $1.00 \times 10^{-4} \div 2.75 \times 10^{-4}$ & 0.0273 & 0.0003 & 0.0020 & 0.0004 & 0.0011 & 0.0016 & 0.0001 \\
\hline $2.75 \times 10^{-4} \div 7.69 \times 10^{-4}$ & 0.0275 & 0.0002 & 0.0020 & 0.0003 & 0.0011 & 0.0017 & 0.0001 \\
\hline $7.69 \times 10^{-4} \div 2.98 \times 10^{-3}$ & 0.0273 & 0.0002 & 0.0020 & 0.0004 & 0.0011 & 0.0016 & 0.0001 \\
\hline $2.98 \times 10^{-3} \div 5.75 \times 10^{-3}$ & 0.0270 & 0.0003 & 0.0020 & 0.0004 & 0.0011 & 0.0016 & 0.0001 \\
\hline $5.75 \times 10^{-3} \div 1.58 \times 10^{-2}$ & 0.0276 & 0.0007 & 0.0021 & 0.0006 & 0.0011 & 0.0017 & 0.0001 \\
\hline
\end{tabular}


Fig. 6 Fraction of DIS events with forward photons with $\eta>7.9$ as a function of $Q^{2}$ and $x_{B j}$ in the kinematic region $6<Q^{2}<100 \mathrm{GeV}^{2}$ and $0.05<y<0.6$. The error bars shows the quadratic sum of the statistical and the uncorrelated systematic uncertainties. The shaded band shows the correlated systematic uncertainties. The expectation from the LEPTO and CDM models are also shown

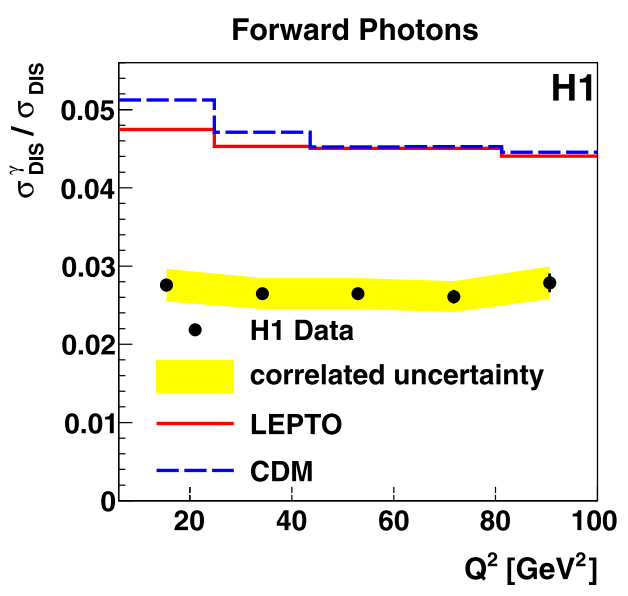

surement also shows significant discrepancies of the predictions of the hadronic interaction models compared to the data.

The measurement of forward photons allows a test of the limiting fragmentation hypothesis, according to which the production of forward photons in DIS is insensitive to $Q^{2}$ and $x_{B j}$. To investigate this prediction, the ratio of the forward photon production cross section to the inclusive DIS cross section is measured as a function of $Q^{2}$ and $x_{B j}$ (Table 2 and Fig. 6). Within the uncertainties the fraction of DIS events with forward photons is independent from $Q^{2}$ and $x_{B j}$ in agreement with the limiting fragmentation hypothesis. A similar conclusion was obtained in the earlier H1 analysis of forward neutron production [7]. The LEPTO and CDM predictions also included in Fig. 6 display a significant difference in normalisation compared to data as well as a slight dependence as a function of $Q^{2}$ and $x_{B j}$.

\section{Summary}

The production of high energy forward photons in the pseudorapidity range $\eta>7.9$ is studied for the first time at HERA in deep-inelastic positron-proton scattering in the kinematic region $6<Q^{2}<100 \mathrm{GeV}^{2}, 0.05<y<0.6$. The normalised DIS cross sections are presented for the production of the most energetic photon as a function of the longitudinal momentum fraction and transverse momentum in the range $0.1<x_{L}^{\text {lead }}<0.7$, and as a function of the sum of longitudinal momentum fractions of all forward photons in the range $0.1<x_{L}^{\text {sum }}<0.95$. The predictions of Monte Carlo models overestimate the rate of photons. The shapes of the measured cross sections are well described by the LEPTO MC simulation, while the colour dipole model predicts harder spectra in $x_{L}$ and $p_{T}$. The measurement is also compared to predictions of models which are commonly used for the simulation of cosmic ray air shower cascades.
All these models predict different spectra in $x_{L}$ and $p_{T}$. None of the models can describe the data in rate and in shape. On average these models overestimate the forward photon production cross section by $30 \%$ to $50 \%$. Within the measured kinematic range, the relative rate of forward photons in DIS events is observed to be independent of $Q^{2}$ and $x_{B j}$, in agreement with the hypothesis of limiting fragmentation. The present measurement provides new information to further improve the understanding of proton fragmentation in collider and cosmic ray experiments.

Acknowledgements We are grateful to the HERA machine group whose outstanding efforts have made this experiment possible. We thank the engineers and technicians for their work in constructing and maintaining the $\mathrm{H} 1$ detector, our funding agencies for financial support, the DESY technical staff for continual assistance and the DESY directorate for support and for the hospitality which they extend to the non-DESY members of the collaboration. We also wish to thank Tanguy Pierog and Ralph Engel for providing the predictions of cosmic ray models.

Open Access This article is distributed under the terms of the Creative Commons Attribution Noncommercial License which permits any noncommercial use, distribution, and reproduction in any medium, provided the original author(s) and source are credited.

\section{References}

1. R. Engel, Nucl. Phys. B, Proc. Suppl. 75A, 62 (1999) [astro-ph/9811225]

2. A. Bunyatyan et al., in Proceedings of the Workshop on the Implications of HERA for LHC Physics, Geneva, Switzerland, 26-30 May 2008 (2008), p. 611 DESY-PROC-2009-02 [arXiv:0903.3861v2]

3. C. Adloff et al. (H1 Collaboration), Eur. Phys. J. C 6, 587 (1999) [hep-ex/9811013]

4. S. Chekanov et al. (ZEUS Collaboration), Nucl. Phys. B 637, 3 (2002) [hep-ex/0205076]

5. S. Chekanov et al. (ZEUS Collaboration), Nucl. Phys. B 776, 1 (2007) [hep-ex/0702028]

6. S. Chekanov et al. (ZEUS Collaboration), J. High Energy Phys. 06, 074 (2009) [arXiv:0812.2416] 
7. F.D. Aaron et al. (H1 Collaboration), Eur. Phys. J. C 68, 381 (2010) [arXiv:1001.0532]

8. J. Benecke et al., Phys. Rev. 188, 2159 (1969)

9. T.T. Chou, C.-N. Yang, Phys. Rev. D 50, 590 (1994)

10. E. Pare et al., Phys. Lett. B 242, 531 (1990)

11. O. Adriani et al. (LHCf Collaboration), Phys. Lett. B 703, 128 (2011) [arXiv:1104.5294]

12. I. Abt et al. (H1 Collaboration), Nucl. Instrum. Methods A 386, 310 (1997)

13. I. Abt et al. (H1 Collaboration), Nucl. Instrum. Methods 386, 348 (1997)

14. R.D. Appuhn et al. (H1 SPACAL Group), Nucl. Instrum. Methods 386, 397 (1997)

15. B. Andrieu et al. (H1 Calorimeter Group), Nucl. Instrum. Methods 336, 460 (1993)

16. T. Nichols et al. (H1 SPACAL Group), Nucl. Instrum. Methods 374, 149 (1996)

17. C. Adloff et al. (H1 Collaboration), Z. Phys. C 76, 613 (1997) [hep-ex/9708016]

18. M. Peez, Search for deviations from the standard model in high transverse energy processes at the electron proton collider HERA. (In French). PhD thesis, Univ. Lyon (2003), CPPM-T2003-04 (available at http://www-h1.desy.de/publications/theses_ list.html)

19. S. Hellwig, Investigation of the $D^{*}-\pi_{\text {slow }}$ double tagging method in charm analyses. (In German). Diploma thesis, Univ. Hamburg (2004) (available at http://www-h1.desy.de/publications/ theses_list.html)

20. D. Pitzl et al., Nucl. Instrum. Methods 454, 334 (2000) [hep-ex/0002044]

21. R. Brun et al., GEANT3, CERN-DD/EE/84-1

22. K. Charchula, G.A. Schuler, H. Spiesberger, DJANGOH 1.4. Comput. Phys. Commun. 81, 381 (1994)
23. G. Ingelman, A. Edin, J. Rathsman, LEPTO 6.5. Comput. Phys. Commun. 101, 108 (1997) [hep-ph/9605286]

24. L. Lönnblad, ARIADNE 4.10. Comput. Phys. Commun. 71, 15 (1992)

25. B. Andersson et al., Phys. Rep. 97, 31 (1983)

26. T. Sjostrand, PYTHIA 5.7 and JETSET 7.4 [hep-ph/9508391]

27. A. Kwiatkowski, H. Spiesberger, H.J. Möhring, HERACLES 1.0. Comput. Phys. Commun. 69, 155 (1992)

28. A. Edin, G. Ingelman, J. Rathsman, Phys. Lett. B 366, 371 (1996) [hep-ph/9508386]

29. A. Aktas et al. (H1 Collaboration), Eur. Phys. J. C 46, 27 (2006) [hep-ex/0508055]

30. F.D. Aaron et al. (H1 Collaboration), Eur. Phys. J. C 64, 561 (2009) [arXiv:0904.3513]

31. K. Werner, F.-M. Liu, T. Pierog, Phys. Rev. C 74, 044902 (2006) [hep-ph/0506232]

32. N.N. Kalmykov, S.S. Ostapchenko, Phys. At. Nucl. 56, 346 (1993)

33. N.N. Kalmykov, S.S. Ostapchenko, A.I. Pavlov, Nucl. Phys. B, Proc. Suppl. 52, 17 (1997)

34. S.S. Ostapchenko, Phys. Rev. D 74, 014026 (2006) [hep-ph/0505259]

35. S.S. Ostapchenko, AIP Conf. Proc. 928, 118 (2007) [arXiv:0706.3784]

36. J. Engel et al., Phys. Rev. D 46, 5013 (1992)

37. V.N. Gribov, Sov. Phys. JETP 26, 414 (1968)

38. P.D.B. Collins, An introduction to Regge theory and high energy physics (Cambridge University Press, Cambridge, 1977)

39. E.-J. Ahn et al., Phys. Rev. D 80, 094003 (2009) [arXiv:0906. 4113]

40. R. Engel, J. Ranft, PHOJET 1.0. Phys. Rev. D 54, 4244 (1996) [hep-ph/9509373] 